\section{$\underset{\substack{\text { hommes } \\ \text { \& migrations }}}{ }$}

\section{Hommes \& migrations}

Revue française de référence sur les dynamiques

migratoires

$1326 \mid 2019$

Londres et ses migrations

\title{
Trois musées face à leur passé colonial : Bruxelles, Amsterdam et Paris
}

\author{
Hélène Bocard
}

\section{(2) OpenEdition \\ Journals}

Édition électronique

URL : https://journals.openedition.org/hommesmigrations/9877

DOI : 10.4000/hommesmigrations.9877

ISSN : 2262-3353

Éditeur

Musée national de l'histoire de l'immigration

Édition imprimée

Date de publication : 1 juillet 2019

Pagination : 174-181

ISBN : 978-2-919040-46-9

ISSN : $1142-852 X$

\section{Référence électronique}

Hélène Bocard, «Trois musées face à leur passé colonial : Bruxelles, Amsterdam et Paris », Hommes \& migrations [En ligne], 1326 | 2019, mis en ligne le 01 juillet 2019, consulté le 15 janvier 2022. URL :

http://journals.openedition.org/hommesmigrations/9877; DOI : https://doi.org/10.4000/

hommesmigrations.9877 


\section{Trois musées face à leur passé colonial : Bruxelles, Amsterdam et Paris}

\author{
Hélène Bocard, conservatrice en chef $d u$ \\ patrimoine au service de la conservation \\ architecturale, Palais de la Porte Dorée.
}

La réouverture en décembre 2018 du musée de Tervuren à Bruxelles, rebaptisé Africa Museum, contribue à questionner la mise en scène de l'histoire coloniale dans l'espace public des anciennes métropoles coloniales. À l'instar de la Belgique, aux Pays-Bas comme en France, d'anciens musées coloniaux, qui ont fait peau neuve à une date plus ou moins récente, affichent une nouvelle identité tout en évoquant leur passé colonial. Inscrites dans l'architecture des bâtiments et les parcours de visites, ces traces coloniales deviennent l'enjeu de nouveaux dispositifs muséographiques.

Nés d'une même volonté de faire connaître l'empire colonial de leur pays à des fins de propagande et d'investissement économique, les anciens musées coloniaux de Bruxelles, Amsterdam et Paris sont également liés par les choix qui ont présidé à la présentation de leurs collections lors de leur ouverture : en effet, le musée de Tervuren (1910) et celui d'Amsterdam (1926) ont inspiré la commission chargée d'organiser le Musée des colonies de Paris après l'Exposition coloniale de 1931. Nous retracerons rapidement l'histoire de chacune de ces trois institutions avant d'en venir à leur physionomie actuelle.

\section{Tervuren : un musée multiple et haut en couleur très imprégné de son passé}

En Belgique, l'idée d'un musée colonial se fait jour en 1894 à l'occasion de l'Exposition internationale d'Anvers. Le projet fut réalisé trois ans plus tard, à l'issue de l'Exposition internationale de 1897, dont le volet colonial se tenait à Tervuren, site d'une ancienne résidence royale dans la campagne bruxelloise. Sur les ruines d'un ancien château, le Palais des colonies fut construit par l'architecte Alfred-Philibert Aldrophe, qui s'inspira du Petit Trianon de Versailles. Il fut ensuite aménagé en

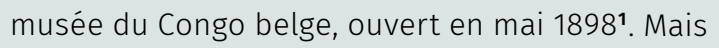

il s'avéra vite exigu et le roi Léopold II décida d'en construire un plus grand pour lequel il fit appel à l'architecte Charles Girault, qui avait édifié à Paris le Musée du Petit Palais pour l'Exposition universelle de 1900. L'inauguration, retardée par le décès du roi, eut lieu le 30 avril 1910. Girault conçut un bâtiment rectangulaire avec une vaste cour au centre et les galeries d'exposition tout autour. Côté jardin, au milieu de la façade longue de 125 mètres, un grand perron ouvre sur une vaste rotonde en marbre couronnée d'une coupole, prolongée de chaque côté par deux larges galeries de marbre. Comme au Petit Palais, le décor est de style classique, sans aucune référence au passé colonial. En revanche, une partie du décor intérieur rappelle l'histoire coloniale, notamment des peintures de paysages du Congo, en partie haute de plusieurs salles de la section d'histoire naturelle ${ }^{2}$. À ce décor peint s'ajoutent des sculptures destinées aux niches de la rotonde centrale (commandes passées dans les années 1910 et 1920 à différents artistes) : allégories autour du thème de l'esclavage et de la mission civilisatrice de la Belgique, figures d'Africains, la Charité et la Justice. Des sculptures chryséléphantines, commandées pour l'exposition de 1897 à divers artistes, furent également installées dans la rotonde et dans d'autres salles. On le voit, le musée était avant tout conçu comme un palais richement décoré, dont les hautes fenêtres laissaient passer une lumière abondante.

Dès sa création, le musée de Tervuren avait une double mission : l'éducation des masses et la propagande des idées coloniales ${ }^{3}$. À son ouverture en 1910, cinq sections le structuraient : économie,

\footnotetext{
1. Sur l'histoire du musée, voir les guides suivants : Baron Alphonse de Haulleville, Le musée du Congo belge à Tervueren, Bruxelles, Imprimerie Veuve Monnom, 1910 ; Joseph Maes, Le musée du Congo belge à Tervueren. Guide illustré du visiteur, Anvers, éd. De Sikkel, 1925 ; Dr Henri Schoudeten, Guide illustré du musée du Congo belge, Bruxelles, Imprimerie Veuve Monnom, 1936 (2e éd.). Voir aussi Maarten Couttenier, 1910-2010. Si les murs pouvaient parler. Le musée de Tervuren, Tervuren, Musée royal de l'Afrique centrale, 2010.

2. Il s'agit de toiles marouflées, réalisées par trois artistes belges (Albert Ciamberlani, Émile Fabry, Omer Dierickx) d'après des photographies. Pour l'ensemble du décor, voir Jacqueline Guisset, Le Congo et l'art belge. 1880-1960, Tervuren/Tournai, Musée royal de l'Afrique centrale/La renaissance du livre, 2003 ; Jean-Luc Vellut (dir.), La mémoire du Congo. Le temps colonial, Tervuren/Gand, Musée royal de l'Afrique centrale, éd. Snoeck, 2005.
}

3. Baron Alphonse de Haulleville, op. cit., pp. 6-7. 


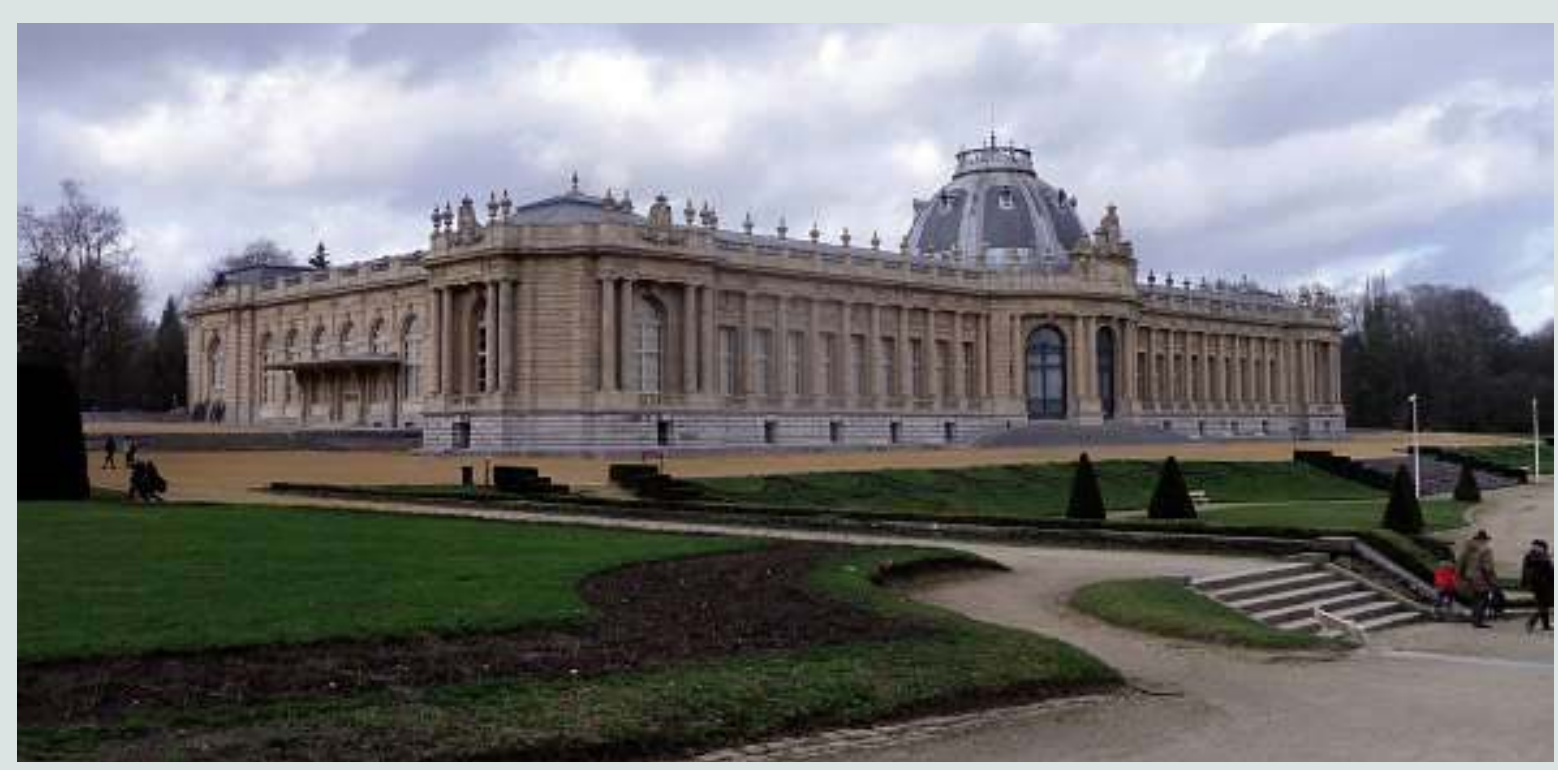

- Vue d’ensemble du Musée de Tervuren, Belgique. Photo Hélène Bocard, 2019. @ Hélène Bocard, Palais de la Porte Dorée.

sciences morales et politiques (l'histoire du Congo et de sa colonisation), ethnographie, sciences naturelles et une dernière intitulée « Photographie et vulgarisation » (qui disparaît ensuite au profit d'une section « Anthropologie et préhistoire »). La section économique mettait l'accent sur les matières premières précieuses provenant de la colonie (le caoutchouc, l'ivoire, le bois et les minerais), afin d'encourager les entreprises et les financiers à investir. Le produit était présenté dans son évolution complète, depuis son état brut jusqu'à sa transformation finale. Une autre partie racontait l'histoire du Congo en mettant l'accent sur les apports de la colonisation (travaux publics, transports, santé, éducation, hygiène) à l'aide de maquettes de villes, de ports, de voies ferrées et d'établissements créés par les colons. La section des sciences naturelles comprenait quatre salles dédiées aux animaux, aux minéraux, à l'entomologie. Dans la section ethnographique, on évoquait aussi bien les modes de vie des populations colonisées (alimentation, chasse, pêche, habitat, etc.) que leur art (fétiches, masques, statues, parures, musique). Pour servir le rôle éducatif du musée et le rendre attractif, on installa des dioramas animaliers (les plus récents ont été conçus dans les années 1970) ou ethnographiques. Charles Girault conçut les vitrines, dont certaines, en forme de pupitres, intègrent des cadres pour photographies permettant de contextualiser les objets exposés.

Très vite, le musée s'est recentré sur trois grands domaines (économie, ethnographie, sciences naturelles) qui le structureront pendant près d'un siècle, même si des mutations ont eu lieu au long des années en fonction des directeurs (ethnologues ou zoologues). Ainsi, la section économique s'est progressivement réduite au profit des galeries ethnographiques; comme dans d'autres musées de la même génération, le statut des objets ethnographiques a évolué en même temps que le regard que l'on porte sur eux, pour atteindre le statut d'œuvres d'art".

Après l'indépendance du Congo, en 1960, le musée changea deux fois de tutelle et devint le Musée royal de l'Afrique centrale, élargissant son aire d'étude au-delà du Congo. Dans les années 1990, se met en place une politique intensive d'expositions temporaires : on mentionnera, en 1995, Trésors cachés du musée de Tervuren, qui présentait 250 chefs-d'œuvre à travers une prestigieuse mise en scène et, en 2005, La mémoire

4. Anne-Marie Bouttiaux, «Des mises en scènes de curiosités aux chefs-d'œuvre mis en scène. Le Musée royal de l'Afrique à Tervuren : un siècle de collections », in Cahiers d'études africaines, $n^{\circ}$ 155-156, 1999, pp. 595-616. 
du Congo. Le temps colonial ${ }^{5}$. Malgré la création d'espaces supplémentaires dès les années 1930 - dont la rénovation du palais des Colonies construit en 1897 -, le palais s'avéra très vite insuffisant et peu fonctionnel, aussi bien pour le travail du personnel que pour la présentation des collections. Au début des années 2000, l'idée de grands travaux s'imposa. En 2010, alors que l'on commémorait le centenaire du musée, le programme de rénovation fut engagé sur l'ensemble du site (7 bâtiments) et le musée ferma en 2013 pour cinq ans.

\section{Entre mise en scène et mise à distance du passé colonial}

En décembre 2018, le musée a rouvert, agrandi d'un nouveau bâtiment destiné à l'accueil du public, à partir duquel on rejoint le palais par un itinéraire souterrain. Avant d'arriver au parcours muséal, on pénètre dans une sorte de sas, dédié à l'histoire des collections du musée. D'un côté, l'histoire des collections et des modes d'acquisition est évoquée à travers textes et objets. De l'autre, une salle où sont parquées derrière une clôture, comme des bêtes de foire, des statues des XIXe et $\mathrm{XX}$ siècles, autrefois exposées dans la section ethnographique. On y voit, par exemple, le groupe des Aniota, dont les personnages étaient revêtus d'habits et de masques en tissu. Caricaturales dans la façon de représenter le colonisé et véhiculant une connotation raciste, elles ont été mises « horsjeu » comme l'explique un texte introductif sur le mur : " De 1908 à 1960, le musée était financé par le ministère des colonies, ce qui se reflétait dans la sélection et la présentation des objets exposés. Le musée joua ainsi un rôle important dans la perception que le public avait de l'Afrique et des Africains, et dans la glorification de la colonie et de ses fondateurs. Aujourd'hui encore, certains aspects de cette perception demeurent vivaces. Les images que l'on voit ici faisaient autrefois partie de l'exposition permanente, mais elles n'y ont plus leur place aujourd'hui. »

À l'issue de ces deux salles, on accède plus loin au palais et au parcours muséal proprement dit. Le musée actuel reprend en partie les grandes sections d'origine. La section « Sciences naturelles » est la plus spectaculaire, avec une grande galerie où trônent, au centre, de grands mammifères naturalisés, et tout autour d'anciennes vitrines « customisées » (habillées de grandes photographies en couleur) abritant pour certaines des dioramas sur la faune et la flore. La salle des crocodiles constitue une sorte de témoignage de l'ancienne muséographie, avec de très belles vitrines - pupitres en bois et cadres pour photographies en partie haute.

La section ethnographie revêt plusieurs formes: deux galeries que l'on qualifiera de traditionnelles, dédiées aux rituels et aux cérémonies ainsi qu'aux langues et aux musiques ; une autre, dénommée Art sans pareil, qui met en scène des statues, des masques, des fétiches admirés pour leurs qualités esthétiques et plastiques, dans l'esprit « arts premiers » du Musée du Quai Branly à Paris. En lieu et place de l'ancienne section "sciences économiques », la galerie dénommée « le paradoxe des ressources » présente, face à face, les ressources et leur exploitation à l'époque coloniale et aujourd'hui. Sur les côtés, ont été conservées les anciennes vitrines alors qu'au centre, sont installés des dispositifs sophistiqués, des écrans, des produits multimédias.

À ces sections qui reprennent plus ou moins celles de l'ancien musée, s'ajoutent de nouveaux espaces : celui intitulé " Paysages et biodiversité » traite de questions environnementales à travers une scénographie spectaculaire, foisonnante, à l'image d'une forêt tropicale, mêlant grandes photographies en couleurs et animaux naturalisés. Ailleurs, la salle Afropea parle de l'immigration africaine en Belgique depuis 1960 (des étudiants issus du Congo, du Rwanda et du Burundi aux demandeurs d'asile d'aujourd'hui). Enfin, la salle « Une longue histoire » est consacrée à la colonisation du Congo, réservant une vitrine à l'articulation entre esclavage et fait colonial.

Dans une des galeries qui longent la cour, un mémorial, conçu entre les deux guerres, rend hommage à 1508 hommes belges morts dans l'État indépendant du Congo entre 1876 et 1908. Pour la réouverture du musée, un hommage a été ajouté à tous les Africains qui sont morts pendant la même période, « victimes directes ou indirectes de l'État indépendant du Congo »; les noms des ouvriers

5. Cette exposition, et le catalogue qui l'accompagnait, voulait inaugurer une nouvelle histoire coloniale en mettant l'accent sur la multiplicité des approches et en s'intéressant à des domaines peu connus (architecture, urbanisme, musique contemporaine). 
africains morts sur le chantier de l'exposition de 1897 sont inscrits sur une vitre, à côté d'une œuvre commandée à l'artiste congolais Freddy Tsimba.

Le musée de Tervuren, on l'a vu, propose plusieurs types de musées en un : à la fois museum combinant histoire naturelle et ethnographie, musée d'arts premiers et musée de société s'intéressant aux questions environnementales et au fait migratoire. Musée aux multiples facettes et à la scénographie spectaculaire, le passé colonial est évoqué de façon diffuse dans plusieurs sections du musée (ressources, sciences naturelles), et plus précisément dans la salle dénommée « Une longue histoire ». L'ancien musée est présent dans les deux salles introductives ainsi que dans le mobilier et le décor d'origine, en grande partie conservés; des documents figurés (cartes postales des anciennes salles) et des cartels explicatifs le rappellent en maints endroits. L'impression générale est que l'ancien musée est toujours là, dissimulé derrière un habillage plus actuel et plus moderne.

\section{Amsterdam: un musée des cultures du monde face à un passé qui dérange}

À Amsterdam, le musée de l'Institut colonial, inauguré en 1926, rebaptisé Tropen Museum (Musée des Tropiques) en 1950, succède à un premier musée colonial ouvert en 1871 à Haarlem, conçu pour encourager la recherche sur les ressources coloniales et le commerce, mais aussi pour instruire le grand public ${ }^{6}$. Le musée de Haarlem s'avéra vite trop étroit et, au début du XXe siècle, on envisagea son transfert à Amsterdam. Le nouveau musée, consacré aux régions tropicales (Indonésie, Suriname, Antilles néerlandaises), était une annexe de l'Institut colonial, qui comprenait trois sections: économie et commerce, ethnologie, hygiène. L'ensemble (institut et musée) fut construit entre 1913 et 1926 par les architectes Johannes Jacobus et Maria Adrianus van Nieukerken, en brique, dans un style historique (néo-renaissance hollandaise) ${ }^{7}$.

Le bâtiment du musée reflète la richesse des colonies : parmi les huit bas-reliefs situés en hauteur sur les façades, quatre représentent la récolte du caoutchouc, du tabac, du sucre, et du riz.

Par ailleurs, quatre autres bas-reliefs portent sur des pratiques religieuses (animisme, hindouisme, christianisme, islam). Ce décor, dû au peintre W. O. J. Nieuwenkamp, est complété par des frises, des sculptures (figures symboliques,

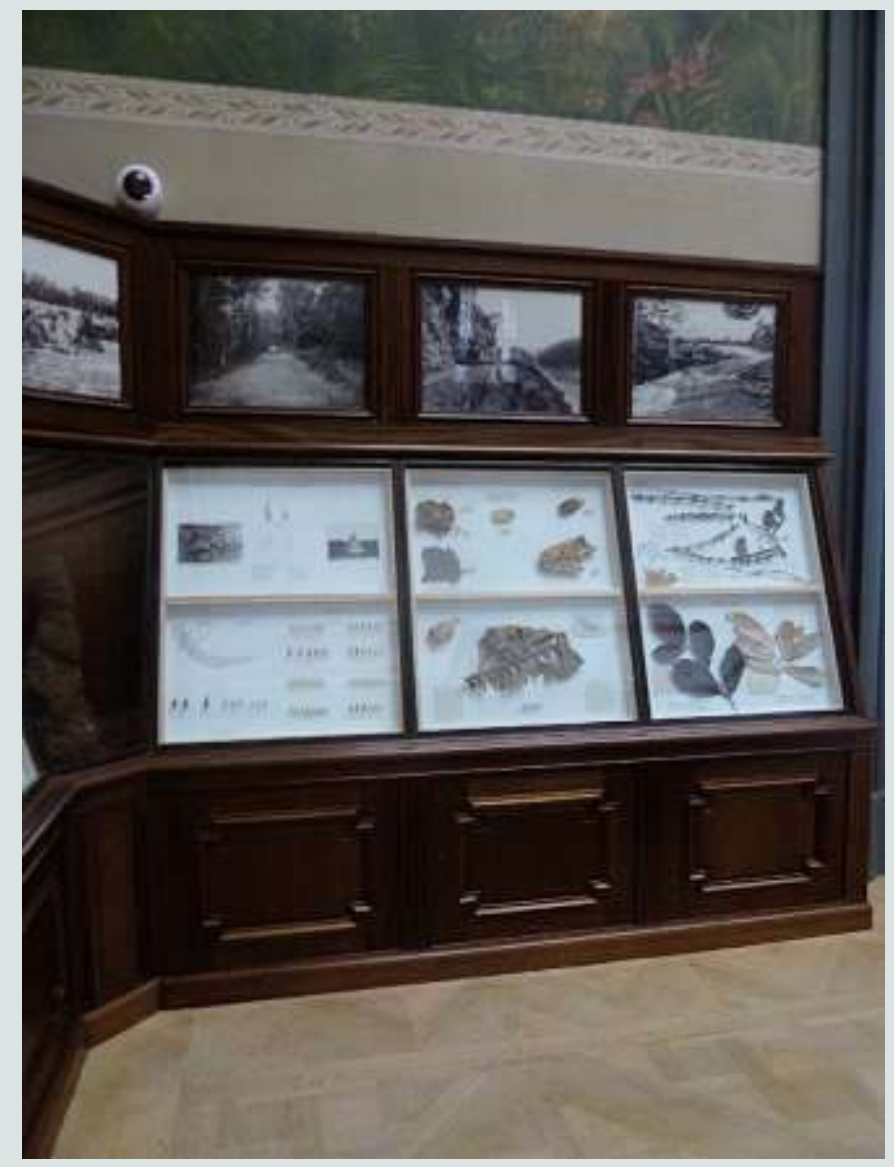

- Vitrines du Musée de Tervuren, Belgique. Photo Hélène Bocard, 2019. @ Hélène Bocard, Palais de la Porte Dorée.

portraits royaux), des ferronneries. Le plan du bâtiment est rectangulaire avec un grand hall central et trois niveaux de galeries marquées par des arcades bicolores et des colonnes aux chapiteaux sculptés. Le décor sculpté évoque des fables indonésiennes ainsi que le premier voyage de Cornelis de Houtman aux Indes orientales; dans les parties hautes, au-dessus des arcades, des scènes peintes par Henk Paulides évoquent encore l'Indonésie. La vue du hall depuis les galeries produit un effet spectaculaire.

6. Il avait été créé par le botaniste F. W. van Eeden, secrétaire de la Société pour la promotion du commerce et de l'industrie aux Pays-Bas ; il ferma en 1923.

7. Voir Tropenmuseum. Museum Guide, Amsterdam, Royal Tropical Museum, 1995 ; Jan Woudsma, An Amsterdam Landmark. The Royal Tropical Institute, Amsterdam, Kit Publishers, 2004. 


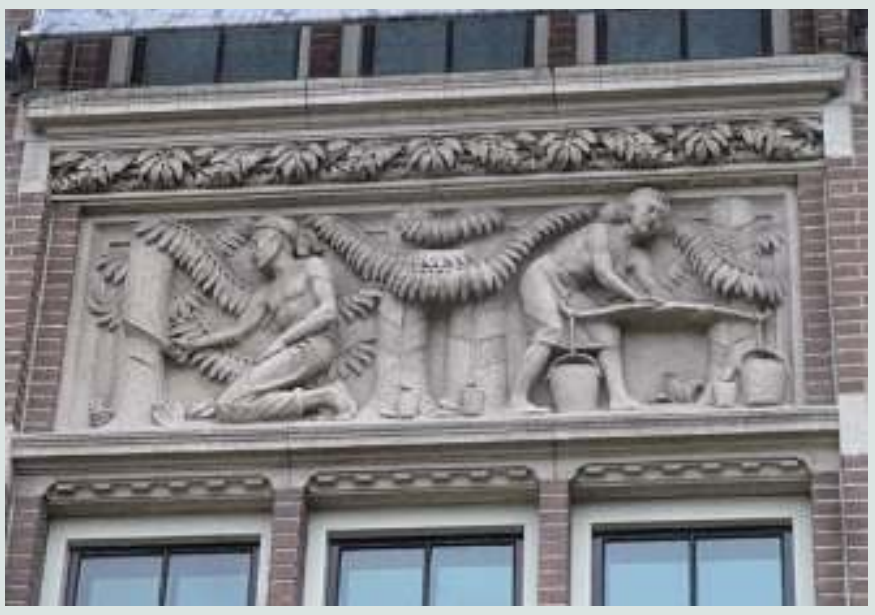

- Bas-relief du Tropen Museum d'Amsterdam,

Pays-Bas. Photo Hélène Bocard, 2019. (c) Hélène Bocard, Palais de la Porte Dorée.

À l'origine, le musée était structuré en trois sections, qui répondaient aux départements scientifiques de l'Institut colonial: dans les galeries, la section ethnographique (objets archéologiques, objets usuels, peintures et sculptures) et la section économique et statistique (produits importés: animaux et végétaux, tissus, ressources minières ; des maquettes et des dioramas); et, plus restreinte, la section de l'hygiène. Le hall était réservé aux expositions temporaires. Le musée fut réaménagé à la fin des années 1970 et dans les années 1990. Comme à Tervuren, la section économique disparut au profit des salles ethnographiques. En 1996, on ouvrit une nouvelle salle sur l'homme et son environnement ; à cette époque, le premier étage était consacré à l'Asie du Sud et du Sud-Est, à l'Océanie, à la musique, à la danse et au théâtre ; au second étage, se trouvaient les collections concernant l'Afrique, l'Amérique du Sud et le Moyen-Orient. On le voit, un tournant avait été pris au moment des indépendances, le musée ne se consacrant plus seulement à l'étude de ses anciennes colonies, mais s'ouvrant au monde entier.

\section{Un musée des cultures du monde}

Actuellement, le Tropen Museum est un musée d'anthropologie et d'ethnographie, décliné sous différentes formes. Au rez-de-chaussée, des expositions conçues pour durer plusieurs années portent sur le monde contemporain : par exemple, l'exposition Things that Matter, installée en 2018 pour une durée de 7 ans, évoque l'importance de certains objets de la vie quotidienne dans le parcours de personnes issues de l'immigration et montre comment ces objets les rattachent à leurs origines ${ }^{8}$. Au premier étage, les galeries d'ethnographie présentent d'un côté, dans l'angle, l'Indonésie, puis, dans les deux grandes galeries, l'Asie du Sud-Est et la Nouvelle-Guinée. Dans les galeries latérales ont été installées une section sur l'histoire coloniale des Pays-Bas, une salle sur le premier musée de Haarlem et, en face, une salle sur l'esclavage. La galerie sur l'histoire coloniale est constituée de maquettes, de mannequins, de dioramas, de panneaux explicatifs. Sept mannequins en plexiglas et mis sous cloches - mis à distance ? - incarnent des personnages réels (un Indonésien et six Européens) ayant vécu aux colonies, dont on nous raconte l'histoire sur des cartels?. Trois autres, réalisés dans les années 1930, représentent un enseignant, un employé de bureau et une couturière. En nous immergeant dans des parcours singuliers, cette muséographie audacieuse, car décalée par rapport aux tendances actuelles, s'avère efficace.

À côté, dans l'angle, l'ancien musée de Haarlem est évoqué dans une salle dont la présentation confine au cabinet de curiosités. On y voit des cartes géographiques, des tableaux, des maquettes de bateaux, des plantes, des meubles en bois précieux, un mannequin représentant le savant à son bureau - le fondateur du musée? -, restituant l'atmosphère d'un bureau de savant ou d'explorateur au milieu du XIXe siècle.

En face, de l'autre côté du hall, toute la galerie est dédiée à l'esclavage, évoqué à travers des portraits (texte et images) d'esclaves ou de personnalités qui y ont pris part ; l'ensemble de témoignages ainsi constitué nous confronte directement à cette histoire et nous questionne sur le rapport que nous entretenons avec elle aujourd'hui. Le niveau supérieur est consacré aux expositions temporaires ${ }^{10}$.

8. Things that Matter questionne des personnes issues de l'immigration sur le sens de certains objets dans leur vie.

9. Par exemple, le peintre Charles Henri Sayers (1901-1943), qui a grandi à Java puis a fait carrière en Europe. II a notamment peint le pavillon hollandais de l'Exposition coloniale de 1931.

10. Au printemps 2019, on pouvait voir une exposition sur le pèlerinage à La Mecque et une autre sur l'influence de la culture visuelle japonaise sur l'Occident. 
Embrassant plusieurs champs

(anthropologique, ethnographique et sociétal), le Tropen Museum se définit aujourd'hui, comme son homologue de Rotterdam, comme un musée des cultures du monde.

\section{Paris : un musée toujours en mutation}

Le Musée des colonies (actuel Palais de la porte Dorée) fut construit à l'occasion de l'Exposition coloniale internationale de 1931. Étant donnée la nécessité de combler le retard par rapport aux pays voisins qui, on l'a vu, possédaient déjà un musée dédié à leurs territoires coloniaux (c'était aussi le cas du Royaume-Uni), le bâtiment construit par Léon Jaussely et Albert Laprade, destiné à survivre à l'exposition, fut très vite baptisé « Musée permanent des colonies ».

Si le palais est plus petit que ses voisins, son plan présente quelques similitudes avec eux : un grand vide central (actuel forum), et deux vides de part et d'autre (hall Marie Curie et auditorium Philippe Dewitte) autour desquels courent deux niveaux de galeries. Sur la rue, un grand hall d'honneur, de près de 100 mètres de long, comparable aux galeries de Tervuren côté jardin. En revanche, il diffère de ses voisins par son décor, proprement monumental et tout à fait exceptionnel dans l'art du XXe siècle : sur la façade, un immense bas-relief réalisé par Alfred Janniot (L'Apport des colonies à la France), dans la salle des fêtes (actuel forum), des fresques par Pierre-Henri Ducos de La Haille et ses associés (L'Apport de la France aux colonies), dans les salons d'apparat, d'autres fresques par Louis Bouquet et les Lemaître (Les Apports artistiques et spirituels de l'Afrique et de l'Asie). Ce décor fait véritablement corps avec le bâtiment, illustrant le renouveau de l'art monumental entre les deux guerres. Il est complété par le pavement en céramique de Gentil et Bourdet sur des dessins d'Albert Laprade, par de nombreux éléments dus aux ferronniers Edgar Brandt, Gilbert Poillerat et Raymond Subes, et par deux très beaux salons dus aux ensembliers Eugène Printz et Jacques-Émile Ruhlmann, de style Art déco.

Dès le mois de septembre 1931, alors que l'Exposition coloniale n'était pas encore terminée, une commission consultative fut chargée de l'élaboration du futur musée et une commission préliminaire fut formée, composée de GeorgesHenri Rivière (artiste, ethnologue, muséographe) ${ }^{11}$,
Albert Laprade, Joseph de la Nézière (artiste peintre ayant vécu au Maroc sous l'administration de Lyautey), Henri Gourdon (gouverneur en Indochine). En octobre, cette commission entreprit un voyage d'études à Amsterdam et à Bruxelles pour visiter les deux musées évoqués plus haut. Le rapport rédigé à l'issue de ce voyage fait l'éloge des deux institutions, en apportant des éléments chiffrés (surfaces des bâtiments, budgets, personnel) et en décrivant les aménagements. La comparaison des superficies dessert le musée parisien $\left(8000 \mathrm{~m}^{2}\right.$ à Tervuren, $7500 \mathrm{~m}^{2}$ à Amsterdam et $5000 \mathrm{~m}^{2}$ à Paris), ce qui fait dire au rapporteur: "Il faudrait, si l'on voulait trouver un pied d'égalité avec ces deux pays colonisateurs, doubler dès maintenant la superficie de ce qui a pu être réalisé à Paris ${ }^{12}$. » Il conclut ensuite: "La Hollande et la Belgique ont fait œuvre admirable. Il serait humiliant que la France fût dans l'impossibilité d'être sur ce terrain, leur égale. Pour cela, il faut avant tout réagir violemment contre certains errements qui depuis longtemps nous déshonorent. Un Musée moderne doit être un organisme vivant et non une maison de retraite pour cacochymes et paresseux ${ }^{13}$. »

Dès le mois de novembre, un premier programme portant sur l'organisation du musée est rédigé et, le 14 décembre, Georges-Henri Rivière remet au gouverneur général Olivier (rapporteur de l'exposition de 1931) un premier budget annuel. Il rejoint ensuite la sous-commission qui évalue les objets figurant à l'inventaire du musée puis, en mai, la commission chargée de l'organisation de la section historique. Le 1er janvier 1932, Gaston Palewski ${ }^{14}$ est officiellement chargé de mission au Musée des colonies, qui ouvre en décembre 1933. Après une fermeture de quelques mois, faute de subventions et de directeur, le musée est placé sous la tutelle de l'Institut d'agronomie générale des Colonies et de la France d'Outre-mer. Il rouvre le 17 janvier 1935 sous le nom de Musée

11. Sur Georges-Henri Rivière, voir le catalogue de l'exposition que le Musée des civilisations de l'Europe et de la Méditerranée (Mucem) lui a consacré en 2018-2019.

12. Ce projet de doubler le bâtiment en profondeur vers le nord, notamment pour accueillir le Musée de la marine, alors à l'étroit dans le Louvre, ne verra finalement jamais le jour.

13. Archives du Musée du Quai Branly.

14. Homme politique ayant travaillé avec Lyautey à Rabat, également licencié en lettres et ancien étudiant de l'École du Louvre. 
de la France d'Outre-mer (FOM). Il comprend trois sections principales: l'Aquarium tropical (histoire naturelle) au rez-de-chaussée inférieur, une section historique au rez-de-chaussée supérieur, une section économique à l'étage. La section historique présentait des bustes, des sculptures, des cartes sur l'histoire coloniale, des Croisades à 1930 ; elle incluait une section « beaux-arts » qui mettait l'accent sur l'influence de l'exotisme sur la vie et l'art français (dont une section consacrée à Paul et Virginie). La section de synthèse (ou section économique) présentait les ressources provenant des colonies. Sur le modèle de Tervuren, elle montrait l'objet importé depuis son état brut jusqu'au produit transformé, à travers différents dispositifs didactiques: dioramas, panneaux rétroéclairés, grandes vitrines, tableaux. Une section dédiée à l'enseignement aux colonies mêlait tableaux statistiques, échelles scolaires, dioramas, maquettes. Enfin, une salle consacrée aux bois coloniaux présentait une soixantaine d'essences de bois exotiques et quelques meubles.

À partir de 1935, le Musée de la FOM, en la personne de son directeur Marius-Ary Leblond ${ }^{15}$, très lié aux cercles artistiques et au marché de l'art, acquit de nombreuses œuvres auprès d'artistes; il développa les salles « beaux-arts » ainsi que les salles d'archéologie et d'art indigène ${ }^{16}$. En 1960, après l'indépendance des pays africains, le Musée de la FOM passe sous la tutelle du nouveau ministère des Affaires culturelles. À l'initiative d'André Malraux, il devient un Musée des arts d'Afrique et d'Océanie (puis Musée des arts africains et océaniens, dit MAAO) structuré en différentes sections correspondant aux aires géographiques: Afrique subsaharienne, Maghreb, Océanie. D'importantes expositions y furent organisées avec, dans les dernières années, une présence forte de l'art contemporain. En janvier 2003, le MAAO ferma ses portes et les collections déménagèrent vers le nouveau Musée du Quai Branly (MQB), s'ajoutant à celles provenant du Musée de l'Homme ${ }^{17}$.

\section{Un lien avec le passé à repenser}

Parmi les différents projets à l'étude, c'est finalement la création d'un Centre de ressources et de mémoires de l'immigration qui a été retenue pour investir le Palais de la Porte Dorée : après une mission de préfiguration mise en place en
2003, la Cité nationale de l'histoire de l'immigration ouvrit en 2007 et devint le Musée national de l'histoire de l'immigration (MNHI) en 2014. II constitue aujourd'hui une des trois directions de l'établissement public du Palais de la Porte Dorée, aux côtés de l'Aquarium tropical et de la direction du bâtiment et de la programmation culturelle. Installé à l'étage, le MNHI propose au visiteur un parcours permanent chrono-thématique (comprenant la Galerie des dons) et un espace dévolu aux expositions temporaires. Relevant du service de la conservation architecturale au sein de la direction du bâtiment, qui a pour mission de valoriser le monument, son décor et son mobilier, le parcours historique situé sur la mezzanine, au-dessus du forum, évoque l'histoire du lieu et des musées successifs. On y voit des objets (un panneau pédagogique de la section de synthèse), des tableaux (dépôts du MQB), des articles de presse, des affiches, etc. Sous sa forme actuelle, ce parcours n'est pas satisfaisant et devra être repensé.

Si les musées d'Amsterdam et de Bruxelles, qui sont aussi des structures de recherche ${ }^{18}$, ont adopté une identité multiple, elles gardent un lien avec leur histoire passée (musées d'ethnographie parlant à la fois des hommes et de leur environnement, collections d'art primitif toujours en place); en revanche, le musée parisien, dépouillé de ses anciennes collections, s'en est clairement éloigné en optant pour un musée de société dédié à une thématique très actuelle, l'immigration.

\section{Conclusion}

Si les trois musées dont il a été question ont engagé leur processus de décolonisation de longue

15. Voir Catherine Fournier, Marius-Ary Leblond, écrivains et critiques d'art, Paris, L'Harmattan, 2001.

16. Une grande partie des peintures que l'on a pu voir récemment dans l'exposition Peintures des lointains, au Musée du Quai Branly-Jacques Chirac, a été acquise à cette époque.

17. Deux ans plus tard, le Musée national des Arts et traditions populaires de Paris ferma ses portes et ses collections déménagèrent à Marseille pour être intégrées au Mucem.

18. Contrairement au souhait exprimé par le rapporteur du voyage d'étude de 1931 dans sa conclusion, le Musée des colonies ne devint jamais un grand établissement scientifique. Ce rôle devait revenir au Musée ethnographique du Trocadéro (devenu en 1938 le Musée de l'Homme), pensé par GeorgesHenri Rivière et Paul Rivet. C'est sans doute ce que comprit rapidement Marius-Ary Leblond, directeur du Musée de la FOM, qui lui donna très vite une forte inflexion artistique. 
date, ils n'en demeurent pas moins, dans l'histoire et le décor des bâtiments qui les abritent, liés à l'histoire coloniale. Nous avons vu de quelle façon leur muséographie actuelle prend en compte cette histoire, ainsi que celle des musées qui les ont précédés. Le Tropen Museum d'Amsterdam est, à notre avis, celui qui regarde avec le plus de lucidité cette histoire qui dérange, innovant dans la muséographie et faisant en plus une place importante au passé esclavagiste de l'Europe.

Le cas parisien est complexe car l'histoire coloniale, beaucoup plus qu'ailleurs, est racontée à travers le bâtiment lui-même et son décor (bas-relief de la façade et fresques du forum). L'articulation de cette histoire avec les missions d'un musée dédié à l'histoire de l'immigration ne va pas de soi ${ }^{19}$. À l'heure actuelle, les supports de communication se rapportant à l'histoire du bâtiment sont insuffisants et différents projets sont à l'étude ${ }^{20}$. Si le site de Tervuren et l'architecture du palais, relativement neutre, ont permis une modernisation intelligente de l'ancien musée en construisant un nouveau bâtiment et en faisant un compromis séduisant entre l'ancienne muséographie et l'actuelle, une telle métamorphose n'est pas envisageable dans le palais parisien. Pour qu'il évolue vers un musée d'histoire qui doit approfondir les relations entre histoire coloniale et histoire de l'immigration, il faut imaginer d'autres formes de réponses aux contradictions que pose un musée de l'histoire de l'immigration dans un bâtiment colonial. On peut déjà imaginer d'accompagner le parcours de visite par un discours clair sur le fait colonial et sur le bâtiment à travers différents types de supports (papier, multimédia). Ensuite, au sein de la programmation culturelle, en dialogue avec les autres directions de l'établissement, on peut proposer une offre autour du palais et de l'histoire coloniale - et de la décolonisation - dont une des conséquences est d'avoir inscrit dans l'imaginaire français des représentations très stéréotypées des populations immigrées en provenance de ces anciens pays colonisés. Plutôt que d'envisager le monument, par ailleurs exceptionnel (pour ne pas dire unique), comme une contrainte, il faut, à notre avis, y voir l'opportunité d'innover en matière muséographique, technologique et culturelle.

19. Le parcours permanent sur l'histoire de l'immigration, dont la refonte est prévue pour 2020, devrait prendre en compte cette articulation.

20. En 2018, une grande peinture représentant un plan de l'exposition coloniale, autrefois dans l'escalier nord, a été accrochée dans le hall d'honneur à côté d'une maquette de l'exposition datant également de 1931 ; c'est là une première étape pour mieux contextualiser le bâtiment et expliquer au visiteur où il se trouve. 